\title{
Entendimento e adesão dos jovens às medidas de restrição social na pandemia de COVID-19
}

\author{
Understanding and adherence of young people to measures of social restrictions of \\ COVID-19 pandemic
}
Comprensión y adherencia de los jóvenes a las medidas de restricción social en la pandemia COVID-19

Thais Gontijo Goulart Leite ${ }^{1 *}$, Sofia Cunha Mafra ${ }^{1}$, Rafael Fagundes dos Anjos Araújo1, Eduardo Mariano de Oliveira Melo e Silva ${ }^{1}$, Ana Maria de Jesus Cardoso'.

\section{RESUMO}

Objetivo: Identificar a compreensão e a adesão da população adulta jovem às medidas de isolamento, bem como a consciência de sua posição enquanto propagadora da doença e de formação de opinião. Métodos: Estudo observacional transversal com aplicação de questionários online via rede social no período de junho a julho de 2020. Resultados: 290 questionários de uma população majoritariamente jovem de 18 a 40 anos, feminina, com alta escolaridade e estratificados pela área de atuação - área da saúde ou não. Foi observada diferença significativa na comparação entre o número de dias em que os indivíduos saíram de casa com o gênero, área de atuação e adesão correta às medidas de isolamento. Houve uma diferença na adesão referida entre as faixas etárias $(p=0,021)$ e um percentual expressivo de indivíduos que se informam por meio de redes sociais $64,8 \%$, não sendo encontrada diferença entre a área de atuação para a fonte de informação utilizada $(p=0,055)$. Conclusão: Bom grau de compreensão é observado quanto à importância das medidas de isolamento social, porém a adesão é inconsistente entre as faixas etárias, o que pode refletir o novo padrão etário de acometimento da onda de infecções em 2021.

Palavras-chave: Isolamento social, Jovens, Saúde pública, COVID-19.

\begin{abstract}
Objective: To identify the understanding and adherence of the young adult population to isolation measures, as well as the awareness of their position as a propagator of the disease and opinion formation. Methods: Cross-sectional observational study with application of online questionnaires via social network from June to July 2020. Results: 290 questionnaires from a mostly young population aged 18 to 40 years, female, with high education and stratified by area of activity - area health or not. A significant difference was observed when comparing the number of days the individuals left home with gender, area of activity and correct adherence to isolation measures. There was a difference in reported adherence between age groups $(p=0.021)$ and a significant percentage of individuals who inform themselves through social networks $64.8 \%$, with no difference being found between the area of expertise for the source of information used $(p=0.055)$. Conclusion: A good degree of understanding is observed regarding the importance of social isolation measures, but adherence is inconsistent between age groups, which may reflect the new age pattern of involvement of the wave of infections in 2021.
\end{abstract}

Keywords: Social isolation, Young people, Public health, COVID-19.

\footnotetext{
${ }^{1}$ Faculdade Ciências Médicas de Minas Gerais (FCMMG), Belo Horizonte - MG.

*E-mail: thaisgoulartleite@gmail.com
} 


\section{RESUMEN}

Objetivo: Identificar la comprensión y adhesión de la población adulta joven a las medidas de aislamiento, así como la conciencia de su posición como propagadora de la enfermedad y formación de opinión. Métodos: Estudio observacional transversal con aplicación de cuestionarios online vía red social de junio a julio de 2020.

Resultados: 290 cuestionarios de una población mayoritariamente joven de 18 a 40 años, del sexo femenino, con educación superior y estratificada por área de actividad - área de salud o no. Se observó una diferencia significativa al comparar el número de días que los individuos abandonaron el hogar con el sexo, el área de actividad y la correcta adherencia a las medidas de aislamiento. Hubo diferencia en la adherencia reportada entre los grupos de edad $(p=0.021)$ y un porcentaje significativo de individuos que se informan a través de las redes sociales $64.8 \%$, no encontrándose diferencia entre el área de especialización por la fuente de información utilizada $(p=0,055)$. Conclusión: Se observa un buen grado de comprensión sobre la importancia de las medidas de aislamiento social, pero la adherencia es inconsistente entre grupos de edad, lo que puede reflejar el patrón de nueva edad de participación de la ola de infecciones en 2021.

Palabras clave: Aislamiento social, Joven, Salud pública, COVID-19.

\section{INTRODUÇÃO}

Em 2020 o mundo foi assolado pela pandemia do novo Coronavírus (SARS-COV-2/COVID-19) que alcançou 185 países em menos de três meses. No Brasil, já são mais de 15 milhões de casos confirmados e 436 mil óbitos (BRASIL, 2021). Estes dados crescem exponencialmente, sobretudo frente à taxa de imunização ainda lenta, e às novas cepas que tem impactado o sistema de saúde por afetarem populações mais jovens. O quadro clínico pode variar desde uma apresentação assintomática ou com sintomas leves de uma síndrome febril, até síndromes respiratórias graves com alta letalidade e exigência de suporte respiratório e unidades de Centro de Terapia Intensiva (CTI) (BRASIL, 2021; ZOU L, et al., 2021).

A maior morbimortalidade e gravidade da doença com necessidade de internação foram incialmente associados às faixas etárias de idosos, (LI, 2020). Todavia, durante novas ondas de infecções em 2021, frente às variantes do SARS-COV-2, como a cepa B.1.1.7, tem-se observado o aumento de infecções e desfechos desfavoráveis em pacientes jovens e inicialmente hígidos, o que tem sido objeto de questionamento de estudos recentes (BELO HORIZONTE, 2021; BROOKMAN S, et al., 2021).

De acordo com dados epidemiológicos de uma capital brasileira, 82,2\% dos óbitos até abril de 2021 ocorreram entre indivíduos acima de 60 anos, $15.3 \%$ entre 40 e 59 anos e $2.3 \%$ entre 20 e 39 anos. Por outro lado, $72 \%$ dos casos confirmados foram de indivíduos com idade entre $20-59$ anos e $18 \%$ de indivíduos de 60 anos ou mais (BELO HORIZONTE, 2021).

Diante do poder e do modo de disseminação do vírus, vários países estabeleceram medidas de restrição social como estratégia preventiva para o controle da pandemia, que em última instância visa a minimizar a sobrecarga do sistema de saúde (LI X, et al., 2020). No entanto, a eficácia de tal medida é diretamente proporcional à aceitação e comprometimento a elas pela sociedade (PAPADODOPOULOS DI, 2020).

Ademais, observa-se a importância da adesão de indivíduos oligossintomáticos e possivelmente por indivíduos assintomáticos às políticas de restrição social (WU Z e MCGOOGAN JF, 2020; ZOU L, et al., 2021; VAN DOREMALEN N, et al., 2020). Neste contexto, discute-se a relevância do indivíduo jovem adulto como agentes de transmissão e conscientização (GUILAMO-RAMOS V, et al., 2020; MONOD M, et al., 2021).

Portanto, o presente trabalho objetiva identificar a compreensão e a adesão da população adulta jovem e 18 a 40 anos às medidas de isolamento, bem como a consciência de sua posição enquanto propagadora da doença e de formação de opinião. Logo, esta pesquisa se justifica por de forma inédita fornecer dados epidemiológicos e sociais para que os órgãos competentes possam traçar planos preventivos direcionados a essa faixa etária específica, sobretudo no contexto da segunda onda frente às festas de final de ano e novas mutações já vigentes em 2021. 


\section{MÉTODOS}

A pesquisa foi realizada a partir de um delineamento observacional transversal, com aplicação de questionários por meio do Google Forms e distribuídos em redes sociais. A dispersão dos questionários se deu após a aprovação da pesquisa pelo CONEP (CAAE: 31003120.9.0000.5134). Ela foi iniciada por estudantes de medicina, a partir de uma capital brasileira. O público-alvo foi limitado a indivíduos com acesso às redes como WhatsApp e Instagram, maiores de 18 anos e que aceitaram o Termo de Consentimento Livre e Esclarecido (TCLE). Para garantir o consentimento, ao clicarem no link da pesquisa o participante era direcionado ao TCLE e só tiveram acesso ao questionário aqueles que consentiram com tal.

Os dados foram coletados entre os dias 02 de junho de 2020 e 29 de julho de 2020. Epidemiologicamente, período que corresponde ao primeiro pico da pandemia segundo dados disponibilizados pela prefeitura. Foi utilizado um questionário composto por vinte e duas perguntas, que foram divididas em seis seções (Quadro 1). A faixa etária utilizada no estudo foi categorizada com base nos achados epidemiológicos de que a maioria dos oligossintomáticos da infecção pela COVID-19 enquadram-se entre 18 a 40; idosos considerados de risco para a infecção são aqueles acima de 60, e uma faixa intermediária entre esses últimos. A profissão foi categorizada como sendo estudante/ profissional da saúde ou não, na tentativa de diminuir o viés de informação.

Quadro 1 - Principais domínios e conteúdo das questões.

\begin{tabular}{|c|c|}
\hline Identificação & Q1. Idade \\
Q2. Gênero \\
Q3. Escolaridade \\
Q4. Profissional da saúde
\end{tabular}

Fonte: Leite TGG, et al., 2021.

O tamanho da amostra levou em consideração as informações de um estudo piloto realizado com 20 participantes aleatórios. Para o intervalo de confiança de $95 \%$, e poder de teste de $80 \%$, seriam necessários 270 questionários.

Para as análises descritivas foram calculadas as frequências absolutas e relativas (\%). O Teste de Qui quadrado foi utilizado para avaliar a correlação de duas variáveis categóricas e para avaliar a correlação entre uma variável categórica e uma contínua, foi utilizado o teste de Spearman, ambos considerando-se significativo valor de $p<0,05$. 


\section{RESULTADOS}

A amostra total incluiu 290 questionários respondidos. Os principais parâmetros descritivos da amostra como idade, gênero, escolaridade e profissão (Tabela 1).

Tabela 1 - Estatística descritiva das variáveis categóricas

\begin{tabular}{lcc}
\hline Variável & $\mathbf{N}$ & $\%$ \\
\hline Idade & 256 & 88,3 \\
\hline Entre 18 e 40 anos & 27 & 9,3 \\
Entre 41 e 59 anos & 7 & 2,4 \\
Maior ou igual a 60 anos & 182 & 62,8 \\
\hline Gênero & 107 & 36,9 \\
\hline Feminino & & \\
Masculino & 133 & 45,9 \\
\hline Profissional ou estudante da área da saúde & 157 & 54,1 \\
\hline Não & & \\
Sim & 10 & 78,4 \\
\hline Escolaridade dos profissionais ou estudantes da área de saúde & 6,4 \\
\hline Médio incompleto & 123 \\
Médio completo & 10 & 1,3 \\
Superior incompleto & 2 & 7,6 \\
Superior completo & 12 & \\
Pós-graduação incompleto & & 5,5 \\
\hline Escolaridade geral da amostra & 16 & 8,3 \\
\hline Médio incompleto & 24 & 57,6 \\
Médio completo & 167 & 13,1 \\
Superior incompleto & 38 \\
Superior completo & 14 & 4,8 \\
Pós-graduação incompleto & 31 & 10,7 \\
Pós-graduação completo & & \\
\hline Font Leite TGG, et al., & & \\
\hline
\end{tabular}

Fonte: Leite TGG, et al., 2021.

\section{Conhecimento sobre SARS-COV-2 e a COVID-19}

Em relação ao meio de transmissão, 94,5\% acreditam na transmissão por meio de superfícies contaminadas, ao passo que a transmissão por meio de gotículas expelidas apenas pela pessoa sintomática foi identificada por $7,2 \%$. Sobre a identificação de um caso suspeito, $87,9 \%$ concordaram com a afirmativa "se eu tiver sintomas de gripe, febre, tosse e mal-estar"; $93,4 \%$ da amostra identificaram a saturação dos serviços de saúde como o motivo para a adoção das medidas.

Sobre as medidas de proteção contra os Sars-Cov-2, 66,9\% da amostra acredita na máscara como meio de proteção eficaz, 72,1\% a higienização das mãos com sabão ou álcool 70\% e 90\% evitar aglomerações.

Quanto à fonte de informação, observou-se um percentual importante para jornal televisivo $(71,7 \%)$ e em segundo lugar para as redes sociais (WhatsApp, Instagram, Facebook, Twitter): 64,8\%; ainda, 30\% relatou informar-se por meio de familiares e amigos. Não foi encontrada diferença na avaliação da correlação entre a fonte de informação e a área de estudo / exercício profissional ( $p=0,055)$. 70,7\% dos indivíduos que não atuam na área da saúde relatou informar-se por meio das redes sociais, enquanto para aqueles da área da saúde o percentual foi de $59,9 \%$. 


\section{Adesão ao isolamento}

O segundo domínio buscou identificar a adesão referida ao isolamento e às medidas sanitárias orientadas pelas autoridades, quais medidas foram adotadas, e se estas já eram utilizadas para proteção contra outros patógenos anteriormente à pandemia do SARS-COV-2.

A adesão correta de acordo com as orientações do Ministério da Saúde foi declarada em $61 \%$ das respostas. O dado foi confrontado com o número de dias por semana que o participante referiu sair de casa: $(50,3 \%)$ dos indivíduos saíam apenas um dia e $(5,5 \%)$ saiam os sete dias da semana. Dentre as atividades que levam as pessoas a sair de casa estão compra de mantimentos $(63,1 \%)$, de medicamentos (32\%), visita a familiares (27\%) e cumprimento de escala de trabalho (16,9\%).

Em relação às medidas preventivas adotadas, $90 \%$ afirmaram o uso de máscara e higiene pessoal, (96\%) a lavagem das mãos ou uso de álcool; (75,5\%) higienização de roupas e sapatos usados na rua; (67,2 \%) higienização de utensílios e pertences, $(64 \%)$ banho ao retornar da rua e $(56 \%)$ a higienização de pertences pessoais como carteira e celular. Frente a todas as medidas citadas, $12 \%$ da amostra já as realizavam de forma rotineira e 43 não adotava nenhuma das medidas antes da pandemia. Não houve diferença significativa entre os grupos de área de atuação quando avaliados os quesitos relacionados à higiene ao chegar da rua $(p=0,483)$.

Acerca da adesão correta ao isolamento, houve diferença entre as faixas etárias $(p=0,021)$. Todos os maiores de 60 anos acreditam ter aderido de forma completa às medidas de isolamento. Por outro lado, a opção discordo totalmente foi composta apenas por jovens de $18-40$ anos (2\% da amostra), e a opção discordo parcialmente com a adesão proposta às medidas por jovens (5,9\%) e pela faixa etária intermediária $(3,7 \%)$.

Foi observada diferença significativa na comparação entre o número de dias em que os indivíduos saíram de casa com o gênero, área de atuação e a adesão correta às medidas de isolamento. Mulheres relataram sair mais vezes que os homens (rho $=0,204$ e $p=0,000$ ). Os profissionais e estudantes de saúde semelhantemente ( $r h o=0,124, p=0,035$ ). Em relação ao relato de adesão quanto às medidas de isolamento, a correlação foi significativa e negativa, ou seja, se o indivíduo referia aderir bem às medidas ele também afirmou sair menos de casa ( $r$ o $=-0,447, p=0,000$ ). Não foi observada diferença significativa entre o número de dias que o participante referiu sair de casa com a faixa etária.

\section{Percepção ao isolamento}

Sobre a percepção dos jovens quanto às medidas de isolamento os resultados indicaram que para $90 \%$ dos entrevistados as políticas de isolamento são medidas necessárias e eficazes; e 3,4\% consideraram tais políticas como medida alarmista e sem cunho científico. $89,7 \%$ dos participantes reconheceram as medidas de isolamento como proteção individual e coletiva e apenas $3,1 \%$ as consideraram como uma alternativa prejudicial à sociedade.

Observou-se também que $21,1 \%$ dos voluntários não tomariam a atitude compatível com as recomendações do Ministério da Saúde (MS) à época da aplicação do questionário frente a uma possível contaminação - 16,3\% relataram que se dirigiriam ao centro de saúde imediatamente, 3,2\% não fariam nada, $1,3 \%$ continuariam a trabalhar normalmente e $0,3 \%$ fariam o teste. Ressalta-se que recomendação preconizada pelo MS era permanecer em casa e buscar aconselhamento remoto em caso de sintomas leves.

Em relação ao entendimento do que as medidas de isolamento representam aos olhos do indivíduo, houve associação significativa $(p=0,012)$ no quesito de não ter opinião formada com gênero, com um percentual maior de homens na alternativa $(13,1 \%)$ em comparação com as mulheres $(2,7 \%)$. Já em relação à escolaridade e à área de atuação (profissional de saúde ou não) não foi encontrada relação significativa ( $p=$ $0,540)$ e $(p=0,924)$ com a percepção das políticas de restrição.

Quanto à compreensão do objetivo das medidas de isolamento social como proteção individual e coletiva, todas responderam acreditar nas medidas como uma alternativa de proteção para si e para terceiros $(p=$ 0,086). 


\section{DISCUSSÃO}

A origem das respostas dos questionários é de uma população predominantemente jovem de 18 a 40 anos, feminina, com alta escolaridade - estudo superior incompleto $57,6 \%$ seguida de superior completo $13,1 \%$ e pós-graduação $10,7 \%$, e com $54,1 \%$ de estudantes ou profissionais da área da saúde. Trata-se, portanto, de um recorte da população brasileira com características específicas determinadas pelos algoritmos das redes sociais, meio em que os questionários foram dispersos (DU E, et al., 2021; GELDSETZER P, 2020) Assim, a análise das respostas, sobretudo aquelas contrárias às recomendações de isolamento social e higiene para prevenção da covid-19 é relevante por retratar a visão de um segmento formador de opinião da população.

Grande parte da amostra estudada ter mostrado conhecimento adequado acerca de meios de transmissão, contaminação, quadro clínico e medidas a serem tomadas. No entanto, mesmo frente a uma amostra com alta escolaridade, ainda se obteve $7,2 \%$ dos indivíduos referindo que a transmissão do vírus ocorria por meio de gotículas expelidas apenas pela pessoa sintomática, e que diante de uma possível contaminação 3,2\% não faria nada e 1,3\% continuariam a trabalhar normalmente. De forma mais relevante, $33,1 \%$ da amostra não referiu o uso da máscara e $27,9 \%$ não referiu a higienização das mãos com álcool $70 \%$ como medidas eficazes de proteção contra o SARS-COV-2.

Também não foi encontrada diferença entre as áreas de atuação quanto à adoção correta das medidas sanitárias de proteção $(p=0,950)$. Uma vez que o grupo de indivíduos estudantes e grupo de profissionais de saúde não obtiveram melhores respostas, duas hipóteses foram criadas. A primeira hipótese pode indicar que os demais indivíduos, mesmo que de outras profissões, estavam bem informados, minimizando o viés da área de atuação. A segunda hipótese diz respeito àqueles indivíduos que não responderam corretamente às questões de acordo com as orientações, o que levanta suspeita quanto à qualidade da informação utilizada por indivíduos dos dois grupos, em especial àquele relacionado à área de saúde, que não apresentou diferencial condizente com a respectiva área técnica.

A não referência do uso de máscara e da higienização das mãos como medidas de prevenção contra COVID-19 deve ser destacada, uma vez que são medidas já preconizadas durante outras epidemias de trato respiratório no controle de infecções hospitalares (MARKEL H, et al., 2007; CHERNOZHVKOV V, et al., 2021) Ademais, um estudo britânico mostrou que as taxas de profissionais de saúde assintomáticos ou sintomáticos que testaram positivo para a COVID-19 diminuíram rapidamente para quase zero durante o lockdown no país, correspondente ao declínio na admissão de pacientes infectados (TREIBEL TA, et al., 2020). Esses dados demonstram então como a adesão às medidas de prevenção impacta diretamente no controle da infecção, e ajudam a evitar que os hospitais se tornem centros da transmissão da SARS-COV-2 (RIVETT L, et al., 2020; CLIFF A e SMALLMAN-RAYNOR M, 2013; NUSSBAUMER-STREIT B, et al., 2020; IACOBUCCI G, 2020).

Quanto à percepção sobre as medidas de isolamento social, o presente estudo encontrou que $89,7 \%$ da amostra as considerou como proteção individual e coletiva e $90 \%$ da amostra as considerou necessárias e eficazes. Entretanto, ressalta-se o dado de que 3,4\% da amostra considerou as medidas de isolamento como alarmistas e sem cunho científico e $3,1 \%$ as consideram como prejudiciais à sociedade. Dado semelhante foi encontrado por Bezerra ACV (2020) de 7,88\% da amostra contrários às medidas implementadas. A revisão da Cochrane neste sentido enfatiza o impacto da adesão nos números de infecções, internações e mortes pela doença de forma que pequenas taxas de não adesão impactam no curso da pandemia (NUSSBAUMERSTREIT B, et al., 2020).

Apesar de todas as faixas etárias concordarem que as medidas de isolamento social representam uma alternativa de proteção individual e coletiva $(p=0,096)$, foi encontrada diferença estatística na resposta quanto ao relato de adesão entre os jovens e aqueles de faixa etária intermediária, que informaram adesão parcial ou discordância quanto à adesão, para àquela de indivíduos mais velhos $(p=0,021)$. Dentre os indivíduos com faixa etário de maior risco para a infecção da COVID-19, todos informaram concordar com a adesão completa às medidas. Esse fenômeno foi encontrado de forma similar pelo estudo de Wilson RF (2020) e de Raza A (2020). 
Os principais estudos que buscaram entender fatores relacionados a não adesão às medidas preventivas recomendadas apontam para os impactos financeiros e psicológicos da pandemia como mostraram os estudos de Bezerra ACV, et al. (2020), Natividade MDS, et al. (2020), Aquino EML, et al. (2020), Praveen SV, et al. 2021; para as mensagens conflitantes entre autoridades científicas e políticas quanto às recomendações acerca das medidas, como mostraram os estudos de Al-Dmour H, et al. (2020), Ataguba AO e Ataguba JE (2020), Raza A (2020), Bezerra ACV, et al.(2020); e para a presença da desinformação em massa e, de forma específica à população jovem, o efeito da influência da pressão entre os grupos sociais e uma menor aversão ao risco (ANDREWS JL, et al., 2020; NIVETTE A, et al., 2020; WILSON RF, et al., 2020).

A adesão ao isolamento pode ter relação com o medo inicial de sofrer prejuízos sociais e, geralmente, está mais presente entre aqueles que aderem às medidas. Desta forma, tanto o medo sendo menor entre aqueles que não aderem às medidas quanto a tendência à saturação da adesão com o passar do tempo são sinais de alerta em um contexto presente e futuro: no presente devido à segunda onda de infecções em diversos países e ao tempo prolongado necessário para que se possa vacinar toda a população e gerar imunidade populacional. Em um contexto futuro a não adesão às medidas é preocupante devido à possível necessidade de instalação de medidas de isolamento social intermitentes frente a picos da doença durante o inverno e às novas cepas, que se mostraram mais virulentas.

Ressalta-se também o impacto psicológico das medidas, que cresce de forma proporcional ao tempo de exposição, sobretudo nos extremos de idade- jovens e idosos. Esse fator pode ser uma variável desfavorável no contexto atual de uma segunda onda, tanto pela necessidade de socialização dos jovens quanto dos impactos psiquiátricos do isolamento de idosos. Este último foi mostrado pelo estudo de Daoust JF (2020), com idosos de 27 países que evidenciou que esta população apesar de ser a mais vulnerável à doença, não está sistematicamente mais responsiva às medidas.

Outra explicação para os dados contrários às orientações das autoridades de saúde encontradas nos questionários diz respeito à desinformação e fake news que durante a pandemia, inclusive, adquiriu a terminologia de infodemia pela OMS. Neste contexto, o percentual expressivo de $64,8 \%$ dos indivíduos na presente amostra que utilizam as redes sociais como fonte de informação e $30 \%$ que se informam por meio de familiares e amigos falam a favor desta hipótese.

Dado similar foi encontrado no estudo de Saud M, et al., (2020) com 67,2\% dos indivíduos utilizando mídias sociais para obter informações médicas sobre o SARS-COV-2. De forma contraditória, a utilização desses meios como fonte de informação não diferiu quanto à escolaridade - $57,6 \%$ da amostra estava cursando ensino superior ou pós-graduação - nem quanto à área de atuação $(p=0,555)$. Como destacado pelo estudo de Galhardi CP (2020), destaca que sete em cada dez brasileiros já acreditam em alguma informação falsa sobre a pandemia e o SARS-COV-2 recebidos pelas redes sociais.

Deve-se atentar, portanto, à população jovem neste contexto, por estar mais presente nas redes sociais e, consequentemente, com maior exposição à desinformação, e principalmente dado o fato de que adesão a intervenções não farmacêuticas durante pandemias é menor quando as pessoas se percebem em risco relativamente baixo da doença. O estudo de Wilson, por exemplo, encontrou um percentual de $38 \%$ de jovens infectados pela SARS-COV-2 de 18 a 24 anos que afirmaram terem atendido a eventos sociais durante seu período de exposição. Outra evidência é o aumento observado no número de infecções após aumento de movimentações pelo país, viagens e aglomerações durante as festas de fim de ano, sobretudo pela população jovem, que inclusive culminou em novos decretos de Lockdown em cidades brasileiras (BELO HORIZONTE, 2021).

Ressalta-se a maior susceptibilidade destes à influência de pares em seu comportamento, sobretudo quanto à exposição a riscos, fato que influencia diretamente a crise atual. Prova disso é o achado de que jovens se sentem confortáveis e contraditoriamente pressionados a não usar máscara e aderir a aglomerações se seus semelhantes o fizerem (WILSON RF, et al., 2020). Essa importância da opinião de semelhantes pode ser indicada também no dado encontrado nesta pesquisa de que $30 \%$ da amostra informase por amigos e familiares (ANDREWS JL, et al., 2020; MONOD M, et al., 2021). 
Neste contexto, as redes sociais podem ser uma arma poderosa para as autoridades competentes científicas e políticas para alcançar, de forma maciça e instantânea, uma grande parte da população, como pode favorecer, inclusive, novos picos da doença consequente à desinformação e ao fenômeno da influência de pares no comportamento humano à adesão às medidas de isolamento e higiene (AL-DMOUR $\mathrm{H}$, et al.,2020; DU E, et al., 2021; NIVETTE A, et al., 2021; SAUD M, et al., 2020). Percebe-se, então, a importância de as medidas de prevenção do vírus serem voltadas à população jovem, principalmente no contexto de novas ondas de infecção do Sars-Cov-2, ao contrário das propagandas iniciais que se voltaram ao isolamento da população idosa (MONOD M, et al., 2021; DAOUST JF, et al., 2020).

Por fim, é importante ressaltar que essa pesquisa foi realizada nos primeiros meses de decreto das medidas de isolamento social. Essa cronologia deve ser destacada uma vez que a duração das medidas não era previsível, o que pode ter influenciado significativamente as respostas relacionadas à adesão. Além disso, trata-se de um estudo realizado a partir de relatos pessoais que podem não apresentar uma correspondência objetiva com a realidade. No entanto, apesar das limitações, destaca-se a importância dos resultados encontrados neste estudo, sobretudo no contexto de novas infecções e aumento de morbidade das novas cepas em pacientes jovens. Assim, é importante a realização de novos estudos para avaliar a adesão às medidas preventivas ao longo de todo o período da pandemia.

\section{CONCLUSÃO}

Os dados obtidos revelam bom grau de entendimento quanto à importância das medidas de isolamento social propostas pelo Ministério da Saúde, apesar de haver declarações não correspondentes a partir da não adesão de alguns segmentos. Destaca-se também a diferença na adesão quanto à faixa etária, a qual se mostrou inferior em indivíduos jovens. Tais dados devem ser levados em consideração frente às novas ondas de infecções de novas cepas potencialmente letais às populações jovens. Vale ressaltar a necessidade de mais pesquisas voltadas acerca da compreensão das medidas de higiene e isolamento com enfoque em outros segmentos sociais.

\section{REFERÊNCIAS}

1. AL-DMOUR $\mathrm{H}$, et al. Influence of social media platforms on public health protection against the COVID-19 pandemic via the mediating effects of public health awareness and behavioral changes: integrated model. Journal of medical Internet research, 2020; 22(8): e19996.

2. ANDREWS JL, et al. Peer influence in adolescence: Public-health implications for COVID-19. Trends in Cognitive Sciences, 2020; 24(8): 585-587.

3. AQUINO EML, et al. Medidas de distanciamento social no controle da pandemia de COVID-19: potenciais impactos e desafios no Brasil. Ciência \& Saúde Coletiva, 2020; 25: 2423-2446.

4. ATAGUBA OA, ATAGUBA JE. Social determinants of health: the role of effective communication in the COVID-19 pandemic in developing countries. Global health action,2020; 13(1): 1788263

5. BELO HORIZONTE. Secretaria Municipal de Saúde. Prefeitura de Belo Horizonte. COVID-19 No $259 / 2021$. 30/4/2021. Boletim Epidemiológico e assistencial, Belo Horizonte, ano 259, 30 abr. 2021. Disponível em: https://prefeitura.pbh.gov.br/sites/default/files/estrutura-degoverno/saude/2021/boletim_epidemiologico_assistencial_259_covid-19_30-04-2021.pdf

6. BEZERRA ACV, et al. Fatores associados ao comportamento da população durante o isolamento social na pandemia de COVID-19. Ciência \& Saúde Coletiva,2020; 25: 2411-2421.

7. BRASIL. Manual do Ministérios de Saúde. 2021. Disponível em: https://www.saude.sc.gov.br/coronavirus/arquivos/Manual_23-10-atualizado.pdf

8. BROOKMAN S, et al. Effect of the new SARS-CoV-2 variant B. 1.1. 7 on children and young people. The Lancet Child \& Adolescent Health,2021; 5(4): e9-e10.

9. CHERNOZHUKOV V, et al. Causal impact of masks, policies, behavior on early covid-19 pandemic in the US. Journal of Econometrics, 2021; 220(1): 23-62.

10. CLIFF A, SMALLMAN-RAYNOR M. Oxford textbook of infectious disease control: a geographical analysis from medieval quarantine to global eradication. Oxford University Press, 2013.

11. DAOUST JF, et al. Elderly people and responses to COVID-19 in 27 Countries. PloS one, 2020; 15(7): e0235590.

12. DU E, et al. How do social media and individual behaviors affect epidemic transmission and control? Science of the Total Environment, 2021; 761: 144114.

13. GALHARDI CP, et al. Fato ou Fake? Uma análise da desinformação frente à pandemia da Covid-19 no Brasil. Ciência \& Saúde Coletiva, 2020; 25: 4201-4210. 
14. GELDSETZER P. Use of rapid online surveys to assess people's perceptions during infectious disease outbreaks: a cross-sectional survey on COVID-19. Journal of medical Internet research, 2020; 22(4): e18790.

15. GUILAMO-RAMOS V, et al. Reconsidering Assumptions of Adolescent and Young Adult Severe Acute Respiratory Syndrome Coronavirus 2 Transmission Dynamics. Clinical Infectious Diseases, 2020.

16. IACOBUCCI G. Covid-19: Doctors sound alarm over hospital transmissions. 2020. 369: m2013

17. IBGE. Instituto Brasileiro de Geografia e Estatística [Internet]. Ibge.gov.br. 2021 [cited 2021 Feb 6]. Available from: https://www.ibge.gov.br

18. LAU H, et al. The positive impact of lockdown in Wuhan on containing the COVID-19 outbreak in China. Journal of travel medicine, 2020; 27(3): taaa037.

19. LI X, et al. Risk factors for severity and mortality in adult COVID-19 inpatients in Wuhan. Journal of Allergy and Clinical Immunology, 2020; 146(1): 110-118.

20. MARKEL H, et al. Nonpharmaceutical interventions implemented by US cities during the 1918-1919 influenza pandemic. Jama, 2007; 298(6): 644-654.

21. MONOD M, et al. Age groups that sustain resurging COVID-19 epidemics in the United States. Science, 2021; $371(6536)$.

22. NATIVIDADE MDS, et al. Distanciamento social e condições de vida na pandemia COVID-19 em Salvador-Bahia, Brasil. Ciência \& Saúde Coletiva, 2020; 25: 3385-3392.

23. NIVETTE A, et al. Non-compliance with COVID-19-related public health measures among young adults in Switzerland: Insights from a longitudinal cohort study. Social science \& medicine, 2021; 268(113370).

24. NOGRADY B, et al. What the data say about asymptomatic COVID infections. Nature, 2020; 587(7835): $534-535$.

25. NUSSBAUMER-STREIT B, et al. Quarantine alone or in combination with other public health measures to control COVID-19: a rapid review. Cochrane Database of Systematic Reviews, 2020; 9.

26. PAPADOPOULOS DI, et al. The impact of lockdown measures on COVID-19: a worldwide comparison. MedRxiv, 2020.

27. PREM K, et al. The effect of control strategies to reduce social mixing on outcomes of the COVID-19 epidemic in Wuhan, China: a modelling study. The Lancet Public Health,2020; 5(5): e261-e270.

28. RAZA A, et al. Role of knowledge, behavior, norms, and e-guidelines in controlling the spread of COVID-19: evidence from Pakistan. Environmental Science and Pollution Research, 2020; 1-17

29. RIVETT L, et al. Screening of healthcare workers for SARS-CoV-2 highlights the role of asymptomatic carriage in COVID-19 transmission. Elife, 2020; 9: e58728.

30. SAUD M, et al. Usage of social media during the pandemic: Seeking support and awareness about COVID-19 through social media platforms. Journal of Public Affairs, 2020; 20(4): e2417.

31. PRAVEEN SV, et al. What concerns Indian general public on second wave of COVID-19? A report on social media opinions. Diabetes \& metabolic syndrome, 2021. 15(3): 829.

32. TREIBEL TA, et al. COVID-19: PCR screening of asymptomatic health-care workers at London hospital. The Lancet, 2020; 395(10237): 1608-1610.

33. VAN DOREMALEN N, et al. Aerosol and surface stability of SARS-CoV-2 as compared with SARS-CoV-1. New England journal of medicine, 2020; 382(16): 1564-1567.

34. WILSON RF, et al. Factors influencing risk for COVID-19 exposure among young adults aged 18-23 yearsWinnebago County, Wisconsin, March-July 2020. Morbidity and Mortality Weekly Report, 2020; 69(41): 1497.

35. WU Z, MCGOOGAN JF. Characteristics of and important lessons from the coronavirus disease 2019 (COVID-19) outbreak in China: summary of a report of 72314 cases from the Chinese Center for Disease Control and Prevention. Jama, 2020; 323(13): 1239-1242.

36. ZOU L, et al. SARS-CoV-2 viral load in upper respiratory specimens of infected patients. New England Journal of medicine,2020; 382(12): 1177-1179. 